\title{
Solar Energy Collection on a Spherical Surface
}

\author{
Kuo-Chi Lin ${ }^{1, *}$, Weiwei Deng ${ }^{1}$, Jing Gu ${ }^{1}$, Chan Ham ${ }^{2}$ \\ ${ }^{1}$ College of Engineering and Computer Science, University of Central Florida, Orlando, 32816, Florida, United States \\ ${ }^{2}$ School of Engineering, Southern Polytechnic State University, Marietta, 30060, Georgia, United States \\ *Corresponding Author: Kurt.Lin@ucf.edu
}

Copyright (C 2014 Horizon Research Publishing All rights reserved.

\begin{abstract}
Solar energy is the most abundant energy source on earth. This paper suggests a new concept of solar collection that prints the solar cells directly onto a spherical surface, or a balloon. The energy collection efficiency of such a spherical surface is analyzed and compared with a conventional flat-panel collector. An experimental apparatus is built to test the solar energy reception from all directions, including the scattering and reflections from various terrain. The results show that the usage of spherical collectors such as solar balloons is a viable approach.
\end{abstract}

Keywords Solar Energy, Spherical Surface Collector, Solar Balloon, Renewable Energy

\section{Introduction}

The Sun is the main energy source of the Earth. All fossil fuels used today are indirect forms of solar energy. Most of the renewable energy sources, such as winds and ocean waves, also exist because of the sun. Therefore, collecting energy directly from the sun is an intuitive approach.

Currently, photovoltaic (PV) based solar panels have been in the commercial-use phase for some time and are available in hardware stores. The history of PV module development started with rigid silicon solar cells. Therefore, flat-panel type solar energy collectors have their technology advantages in manufacturing and installation.

However, in city or residential areas, the flat-panel solar collector has its limitations. For examples, the solar panels should always face true south if they are in the northern hemisphere. But the space for an ideal installation may not always be available. Even if they can face the right direction, nearby higher buildings may block the sunrays from that direction. Additionally, the sun's angles in the sky vary according to the days in the year and the inclination of the panel can only be made optimal in certain times during the year. To most solar collectors, the sun-tracking system is too expensive and impractical, and some cannot even adjust their inclinations manually.

A new idea can solve the above problems: forming the solar cells onto a spherical thin shell and enveloping it around a balloon. When the balloon is raised to a certain height, it will not be blocked by the surrounding buildings and can receive the sunrays from all directions in all seasons.

The term "solar balloon" has two different meanings. One is a balloon that absorbs solar energy to heat the air inside the balloon and increase its buoyancy. It is just one type of the hot-air balloons and it has a long history. Recently, its usage has been expanded to space missions [1-4]. The second definition of solar balloon refers to a balloon that is used to collect solar energy for other applications. This type of balloon is also called a "solar collector".

One example of a solar collector is the solar turbine suggested by Papageorgiou-using a floating "solar chimney" to generate hot air and drive a turbine [5]. Another concept has been developed by Cool Earth Solar [6] that uses a large balloon with one side clear so that the sunray can enter the shiny inside of the balloon serving as a mirror that focuses the solar energy to a photovoltaic cell, thus generating electric power.

This paper suggests a new concept of solar collector that wraps solar cells around a balloon, or "prints" the solar cells directly on a surface of the balloon. This approach increases the areas of solar energy collection efficiency, compared with the solar balloon concepts discussed above.

The feasibility of direct printing of solar cells is ensured by the rapid progress in discovering new semiconducting polymers and the development of polymer photovoltaic devices. Polymer solar cells (PSCs) are a light weight, flexible, and potentially low cost form of organic photovoltaics (OPV). The energy conversion efficiency of OPV has increased steadily, with the record cell efficiency reaching 12\% [7]. PSCs have followed a similar trend towards increased performance. Two major factors have been instrumental to the steady increase of efficiency in PSCs [8-14], namely, the bulk heterojunction interpenetrating network and development of novel low band gap materials [15-17]. These newly discovered semiconducting polymers can cover a wider range of the solar spectrum, have improved interchain ordering, and possess increased stability at elevated temperatures. Most importantly, the liquid phase of such polymer solutions enable a wide range of processing methods such as screen printing [18], pad printing [19], inkjet printing [20; 21], 
spray coating [22], and electrospray printing [23]. These new methods make it possible to fabricate flexible, stretchable, and low cost solar cells on curved surfaces.

The organization of this paper is as follows. A formula is introduced to analyze the solar energy collection rate. The efficiencies of solar energy collection by a flat surface and a spherical surface are compared. An experimental setup to analyze the scattered solar energy is described. The efficiency of the spherical-surface collector with the scattered energy added on is discussed. The advantages of a spherical-surface solar energy collector are summarized in the conclusion.

\section{Solar Energy Collection Rate}

The formula for the solar energy collection can be presented by the following equation:

$$
P=I \cdot R_{a b} \cdot A \cdot C_{i n} \cdot C_{c l}
$$

where

$$
\begin{array}{ll}
P: & \text { Power absorbed, Watts } \\
I: & \text { Solar intensity, } \mathrm{W} / \mathrm{m}^{2} \\
R_{a b}: & \text { Solar voltaic absorption rate } \\
A: & \text { Collection area, } \mathrm{m}^{2} \\
C_{i n}: & \text { Coefficient of incident }=\cos \Xi,
\end{array}
$$

where? is the incident angle

$C_{c l}$ : Coefficient of clarity, between 0 and 1 , where

complete occlusion $C_{c l}=0$,

completely clear $C_{c l}=1$.

In our discussion below, $I, R_{a b}$, and $A$ are assumed the same for both spherical and flat-panel collectors.

\section{Spherical-Surface Solar Collector}

The spherical coordinate system $(r, \theta, \phi)$ is used, where the radius $r$ is a constant, $\theta$ is the azimuthal angle in the $x-y$ plane, and $\phi$ is the zenith angle from the positive $z$-axis, as shown in Figure 1.

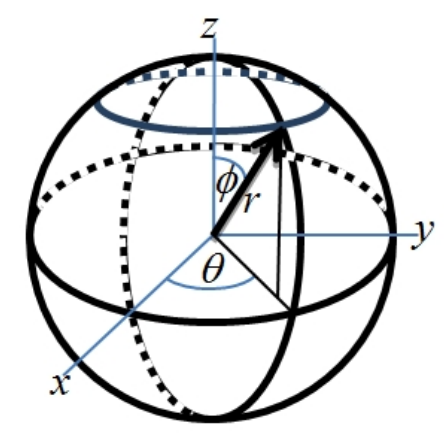

Figure 1. Spherical Coordinate System

At any point on the spherical surface, the unit normal vector is given by $\mathrm{n} n=(\cos \theta \sin \phi) \mathrm{i}+(\sin \theta \sin \phi) \mathrm{j}+(\sin \phi) \mathrm{k}$, where $(\mathrm{i}, \mathrm{j}, \mathrm{k})$ are the unit vectors of the $x-y-z$ coordinate system.
Because of the symmetry of a sphere, the sunrays can come in any direction and will not affect the results. Assuming the sunrays come in along the $x$-axis, the coefficient of incident at $(r, \theta, \phi)$ is given by

$$
\cos \lambda=\cos \theta \sin \phi
$$

Only one side of the sphere, $\theta$ between $-\pi / 2$ and $\pi / 2$, has direct sunrays. Taking the differential area $d A=$ $r^{2}(\sin \phi) d \theta d \phi$ at $(r, \theta, \phi)$,

$$
\begin{aligned}
& \int_{A} \cos \lambda d A=r^{2} \int_{0}^{\pi} \int_{-\pi / 2}^{\pi / 2} \sin ^{2} \varphi \cos \theta d \theta d \varphi \\
& =2 r^{2} \int_{0}^{\pi} \sin ^{2} \varphi d \varphi=r^{2}[\varphi-\sin 2 \varphi / 2]_{0}^{\pi}=\pi r^{2}
\end{aligned}
$$

In the above integration, the azimuthal angle $\theta$ varies only from $-\pi / 2$ to $-\pi / 2$, because the back side of the sphere cannot receive the sunrays.

If the sphere is high enough in the sky, we can assume no occlusion, or $C_{c l}=1$. The solar power absorbed is given by

$$
P_{s p}=\left(I \cdot R_{a b}\right) \cdot \pi r^{2}
$$

\section{Flat-Panel Solar Collector}

For the flat-panel collector, the sunrays incident angle depends on the relative orientation between the Sun and the panel. Figure 2 shows the Sun's paths on the first day of Spring, Summer, and Winter, respectively, in the northern hemisphere. The Sun's angles in the sky include the angle above the horizon depending on the time in a day, and the azimuth (measure from the South) of the Sun depending on the day in a year. There are procedures to obtain the exact sun angles [24]. For simplification, the following analysis use certain average values.

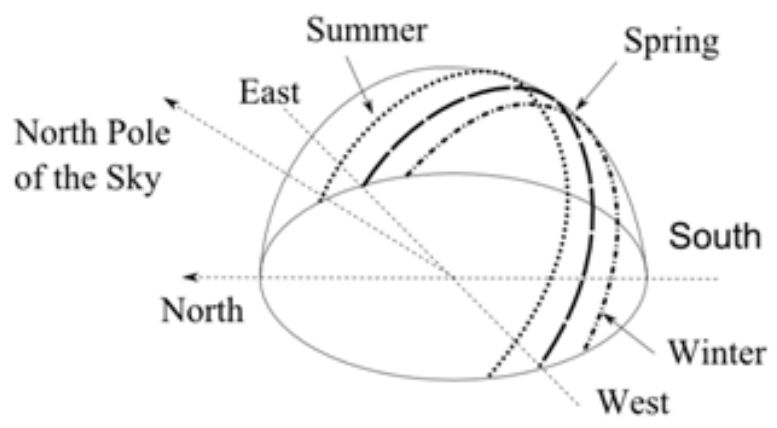

Figure 2. Sun paths for a location in the Northern Hemisphere

Figure 3 shows the sun's paths in the sky both in a day and in a year relative to a flat-panel collector, which is squarely facing the sun at noon of the first day of Spring and Fall. On the first day of Spring, the maximum possible incident angle variations are from $-\pi / 2$ to $\pi / 2$. Therefore, the average coefficient of incident angle in a day is

$$
C_{i n}=\frac{1}{\pi} \int_{-\frac{\pi}{2}}^{\frac{\pi}{2}} \cos \lambda d \lambda=\frac{2}{\pi}
$$




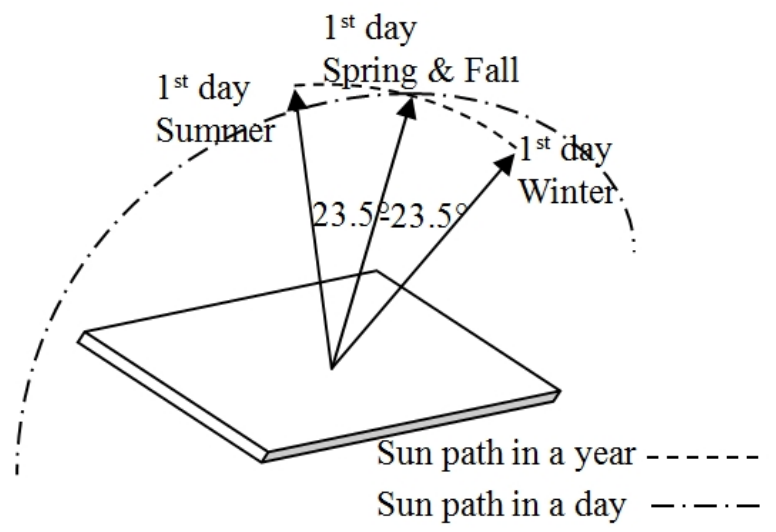

Figure 3. Sun paths a day and in a year

Assuming the flat panel has the same area as the sphere, i.e. $\mathrm{A}=4 \pi r^{2}$, the average power absorbed during the first day of Spring (or Fall) is given by

$$
P_{f}=\left(I \cdot R_{a b}\right)\left(8 C_{c l}\right) r^{2}
$$

Comparing Equation (5) and (6), it seems that the flat-panel collector has a better power absorption efficiency than the spherical-surface collector as long as the coefficient of clarity, $C_{c l}$, is greater than $\pi / 8=39.3 \%$. However, the assumption that the incident angle has a range of $-\pi / 2$ to $\pi / 2$ in a day is an over-estimation. Most flat solar panels will be exposed to the sunrays only when the Sun is relatively high above the horizon. On the other hand, if the balloon is high enough in the sky, the spherical-surface collector it carries will be exposed to the sunrays longer on both ends of the day.

Another factor is that the flat-panel collector efficiency is subject to seasonal adjustments due to the sun's azimuth changes. As shown in Figure 3, during the year the sun's angle changes 23.5 degrees in each direction from the vertical position. Since $\cos \left(23.5^{\circ}\right)=91.7 \%$, it gives the maximum variation of $\mathrm{C}_{\text {in }}$ due to the change of seasons as $9.3 \%$. On the other hand, the spherical surface efficiency is the same no matter what the season is.

\section{Experimental Apparatus}

Equation (4) is derived based on the fact that the back side of the sphere-surface collector is not exposed to direct sunrays. However, the back side can absorb energy from the scattered solar radiation and reflections of sunrays off buildings, terrain, and other surfaces. An experimental apparatus, as shown in Figure 4, has been designed and built to determine the amount of the energy absorbed by the back side of the sphere-surface collector.

The apparatus in Figure 4 consists of a light source inside an oversized hood with a Fresnel lens system, as shown in Figure 5. The lens system is used to minimize the light gradient and create parallel beams like those of the sun. A motor-driven motion mechanism is put in to rotate the hood and simulate the sunrays from various angles during different hours in a day. The design drawing of the motion mechanism is shown in Figure 6.

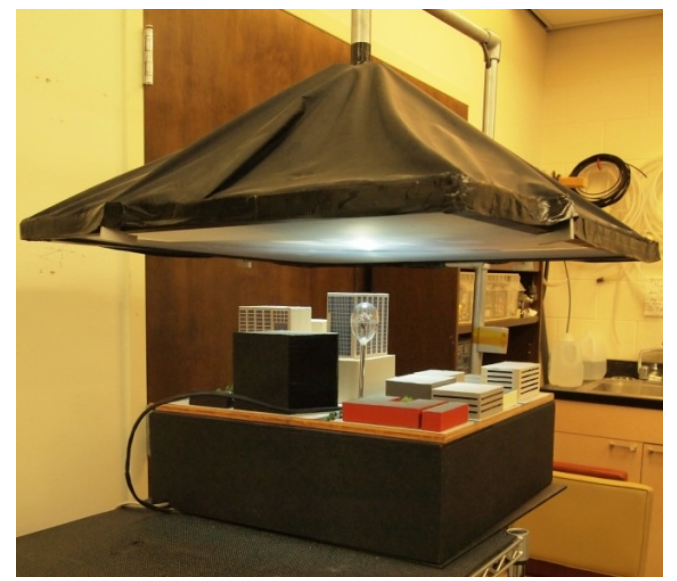

Figure 4. Apparatus measuring lights from all directions

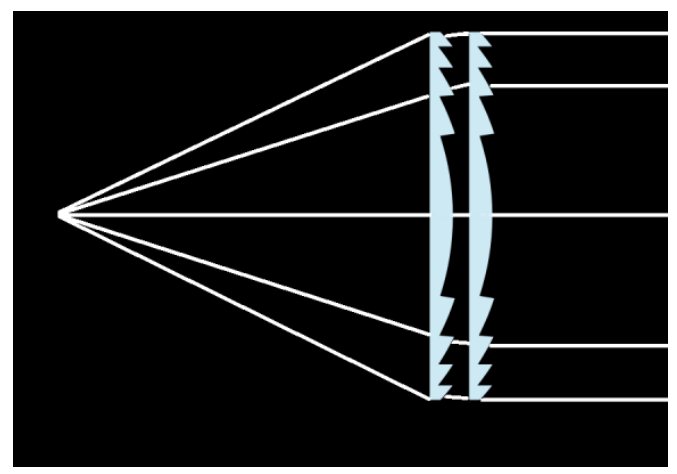

Figure 5. Fresnel Lens

Under the hood, a transparent plastic ball with an octahedron at the center is supported by a post standing on the platform. Each surface of the octahedron has a miniature solar cell soldered on it, as shown in Figure 7. This system represents a miniature solar balloon and the solar cells serve as the sensors to measure the solar energy from all directions. The reason to use an octahedron is that it is easier to make than a true sphere. The solar cells facing the sun (light source) measure the solar energy directly from the sun, while other solar cells on the back measure the scattered and reflected solar energies.

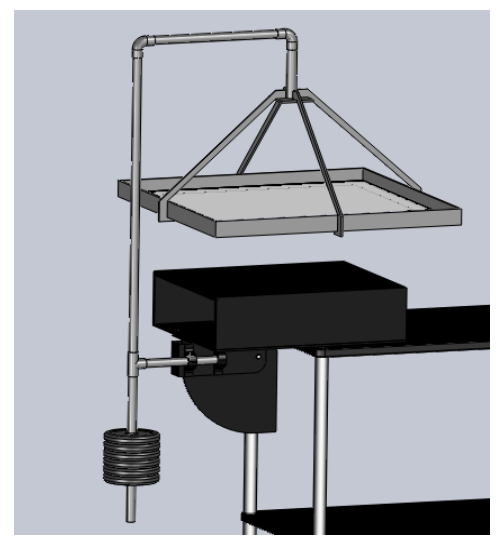

Figure 6. Mechanism to rotate the hood 


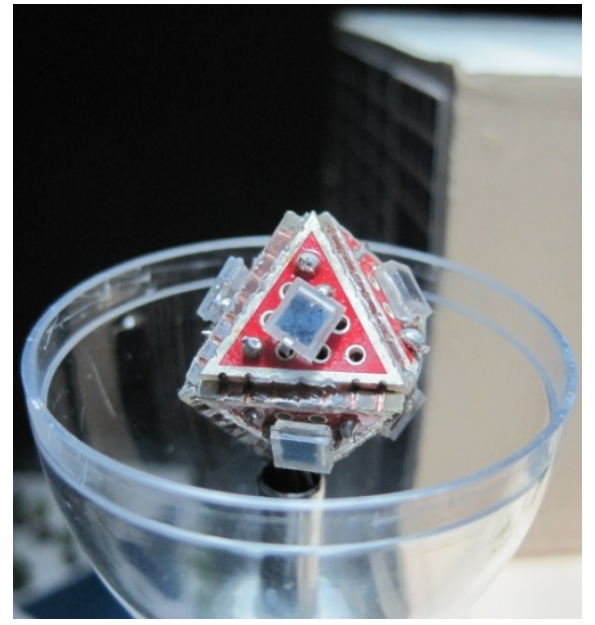

Figure 7. Octahedron with solar sensors

\section{Data Collections}

On the platform, the balloon model is surrounded by a terrain mockup that can reflect light. The hood is rotating from one side to another to simulate the Sun's locations at different hours in the day. Figure 8 shows a mirror that can completely reflect light. Figure 9 shows the readings of all 8 solar cells' outputs, with Sun's angle as the horizontal axis, having similar readings as expected.

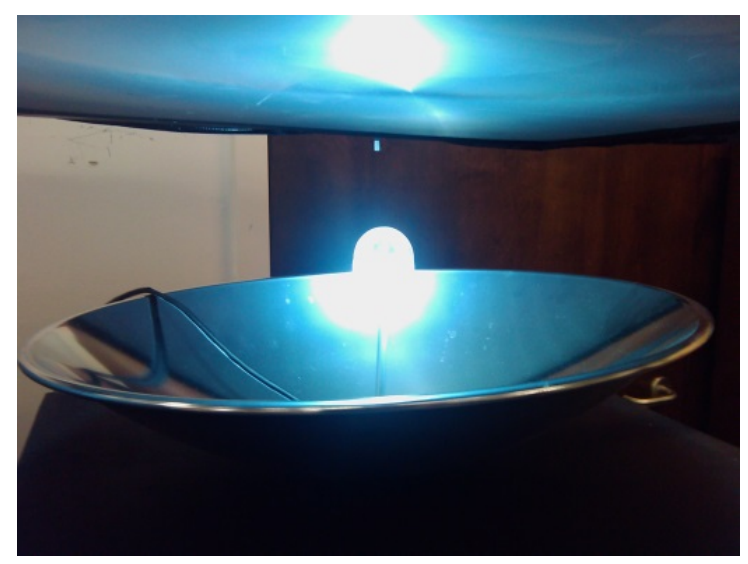

Figure 8. Mirror reflector

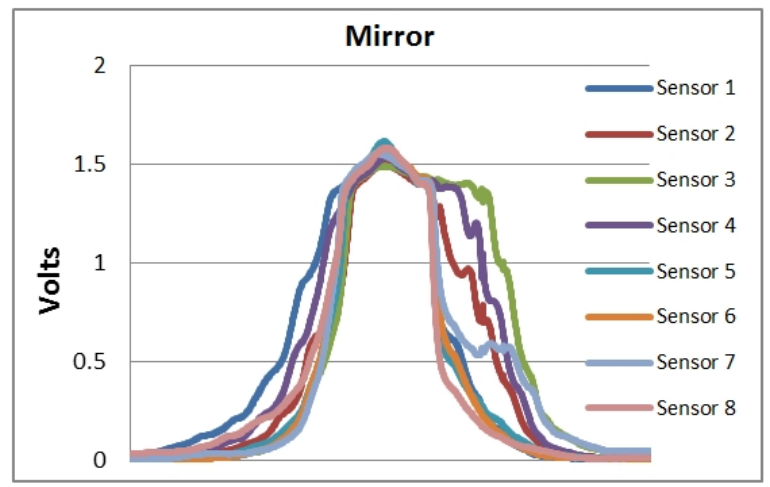

Figure 9. Solar cell readings for mirror reflector
Several different terrain mockups are built to represent the typical city makeup. Figure10 shows a small model of a lake/island park. Figure 11 shows the readings from each solar cell at each Sun's angle. Four of these have higher values representing the four solar cells on the upper faces of the octahedron, which absorb solar energy directly from the sun. On the other hand, the four lower curves are the readings from the solar cells on the lower faces of the octahedron, which absorb the reflective and scattered solar energy.

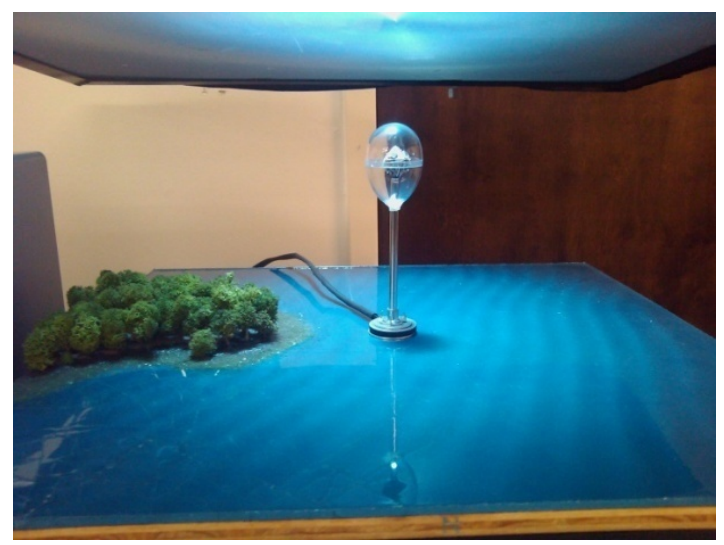

Figure 10. Mockups of a lake park

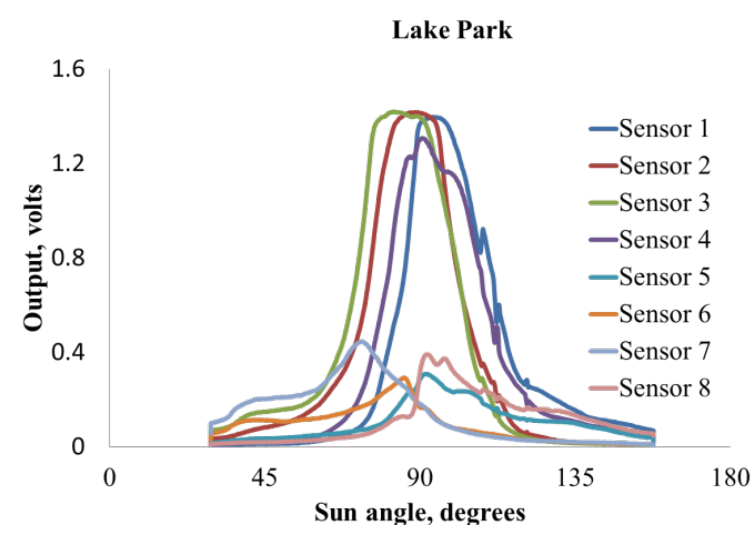

Figure 11. Solar cell readings for a lake park

A small model downtown Orlando mock up is used to measure the scattered radiation energy reflected from the surrounding buildings. Figure 12 is a bird-eye view of downtown Orlando and the letters $a \sim e$ are the five positions of the solar cells.

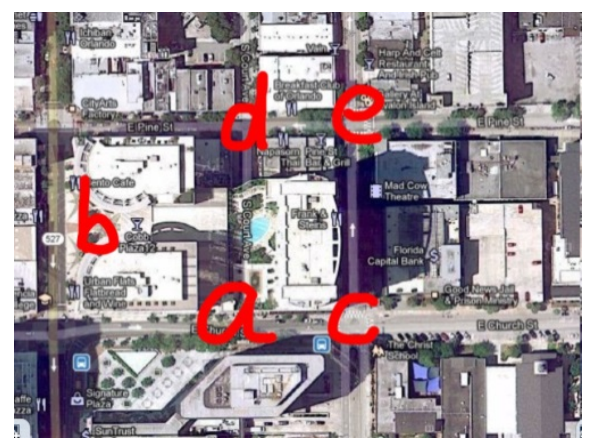

Figure 12. Five solar cell positions in downtown Orlando 
Figures 13 17 show the solar cells at position $a \sim e$, respectively, in Figure 12, and Figure 18 22 show the readings from each solar cell at each Sun's angle.

From the data taken in Figure 18 22, the energy absorbed from the scattered radiation and environmental reflections can be between $25 \% \sim 40 \%$ of the energy from direct sunrays. Adding that extra energy absorbed, the spherical-surface collector performs at the same level as a flat-panel collector with the same surface area.

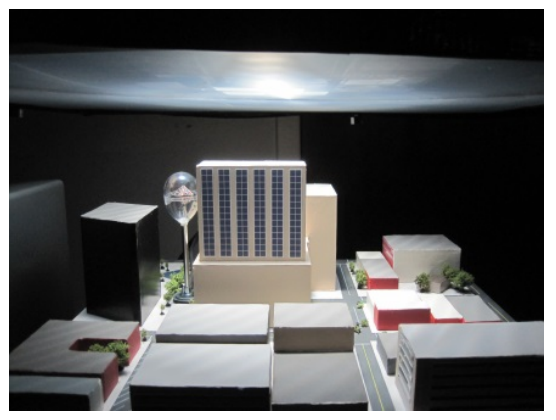

Figure 13. Solar cells at position $a$ in downtown Orlando

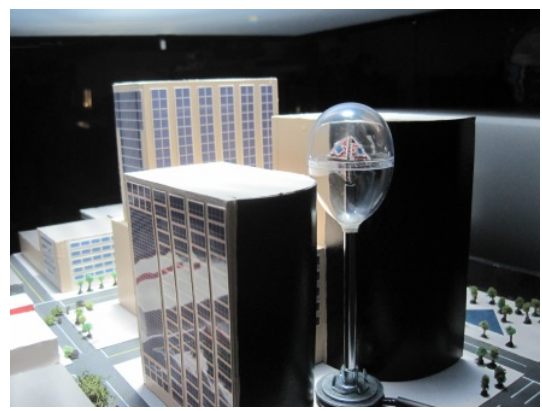

Figure 14. Solar cells at position $b$ in downtown Orlando

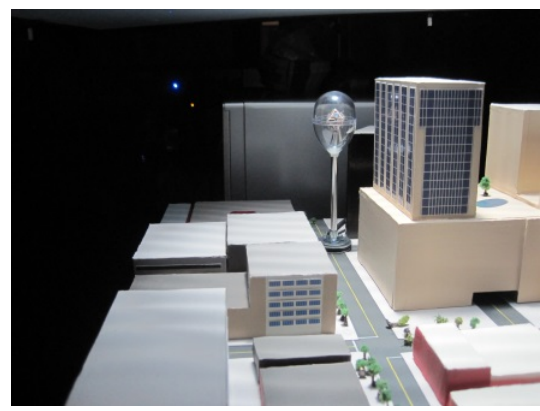

Figure 15. Solar cells at position $c$ in downtown Orlando

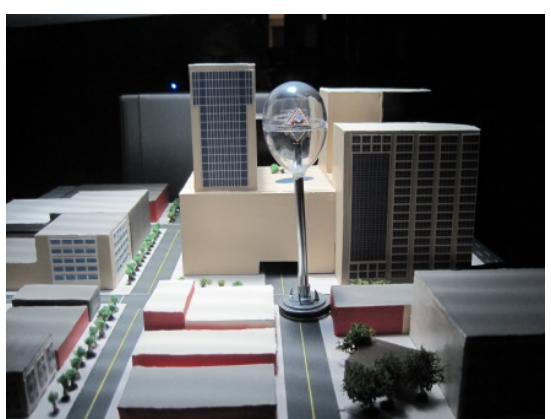

Figure 16. Solar cells at position $d$ in downtown Orlando

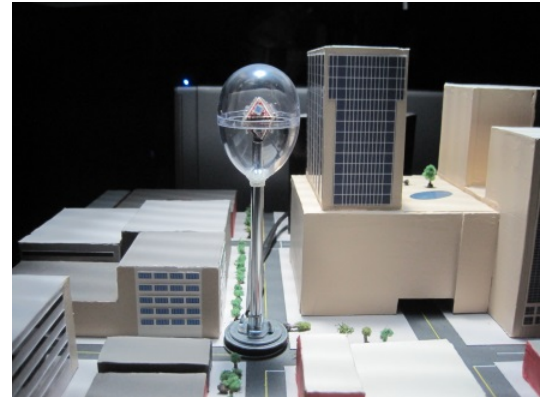

Figure 17. Solar cells at position $e$ in downtown Orlando

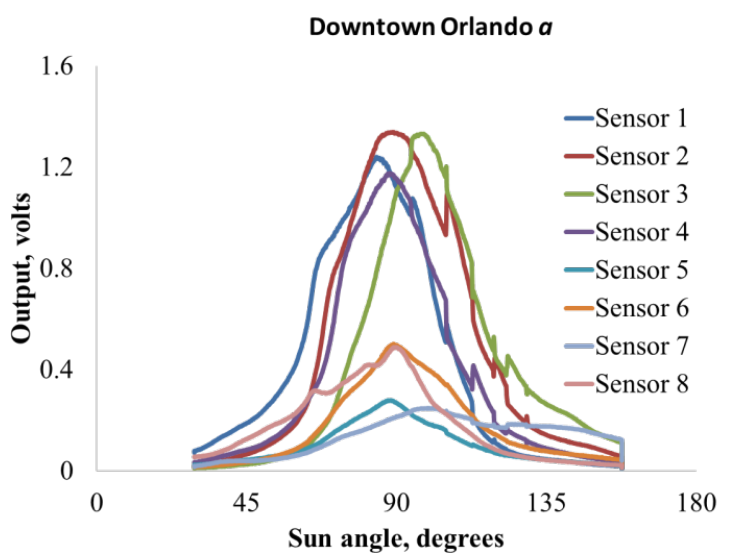

Figure 18. Solar cell readings for position $a$ downtown Orlando

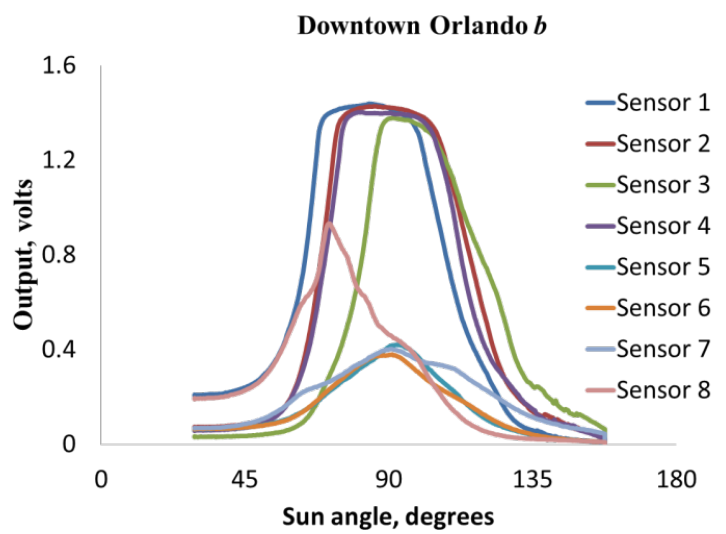

Figure 19. Solar cell readings for position $b$ downtown Orlando

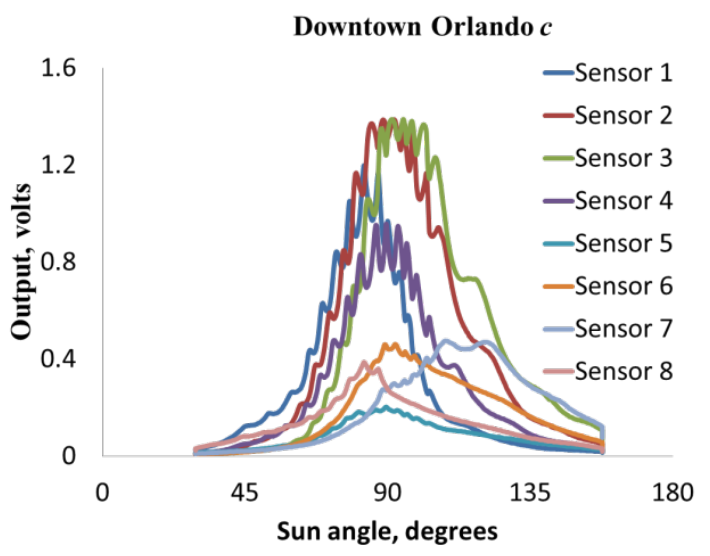

Figure 20. Solar cell readings for position $c$ downtown Orlando 


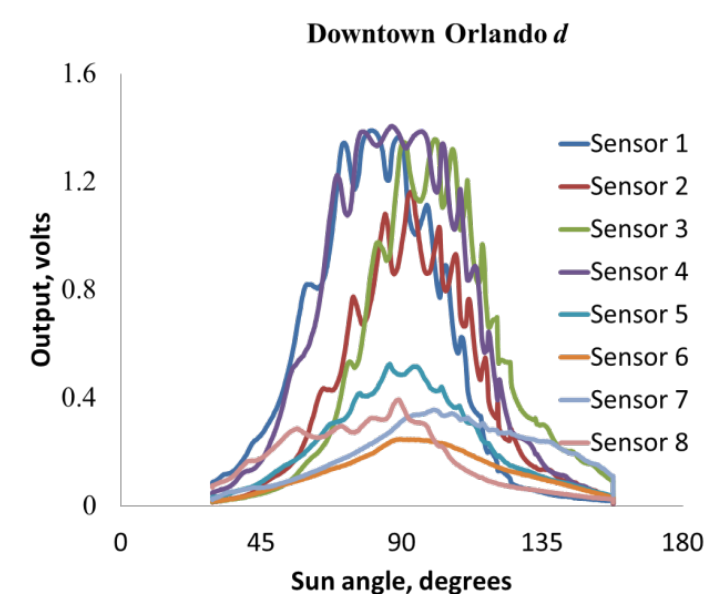

Figure 21. Solar cell readings for position $d$ downtown Orlando

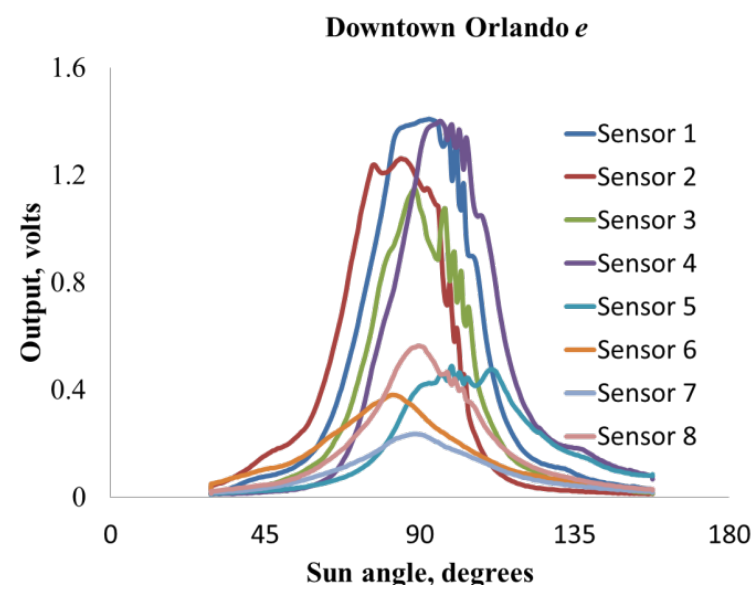

Figure 22. Solar cell readings for position $e$ downtown Orlando

\section{Conclusions}

Based on the above analysis, the following conclusions can be reached:

1. With no occlusion, the flat-panel collector is more efficient than the spherical-surface collector by a factor of more than 2, which can be seen by comparing Equations (4) and (6).

2. Due to the seasonal changes of the sun position in the sky and only a shorter time in a day that the flat-panel collector can see the sun, the advantage of the flat-panel collector is not that much.

3. The back-side of the spherical surface can collect significant amount of scattered radiation and reflected solar energy that makes it as efficient as the flat panel without occlusion.

4. Considering the occlusion factor, the spherical-surface collector can be the better choice in certain situations.

Besides the efficiency consideration, the balloons coated with the PV solar collectors have other features that make them attractive. For example:

1. It is light and portable.

2. It is easier to be taken in bad weather.
3. It can be decorated and made a beautiful scene.

When the PV-painting technology is mature, the new solar balloons should be promoted.

\section{Acknowledgements}

This research is partially sponsored by Duke Energy.

\section{REFERENCES}

[1] G. Aliasi,G. Mengali, A.Quarta. Passive control feasibility of collinear equilibrium points with solar balloons. Journal of Guidance, Control, and Dynamics. 2012;35(5):1657 - 61.

[2] J. Biggs, C. Mcinnes. Passive Orbit Control for Space-Based Geo-Engineering. Journal of Guidance, Control, and Dynamics. 2010;33(3):1017 - 20.

[3] J.Blamont, J. Jones. A new method for landing on Mars. ActaAstronautica. 2002;51(10):723 - 6.

[4] J. Jones, J. Wu. Solar Montgolfiere balloons for Mars. International Balloon Technology Conference. Paper No. AIAA-99-3852; 1999.

[5] C. Papageorgiou. Floating Solar Chimney versus Concrete Solar Chimney Power Plants. Clean Electrical Power, 2007 ICCEP '07 International Conference on; 2007 21-23 May 2007.

[6] Online

Available:http://www.causecast.com/blog/bid/146786/CoolSolar-Earth-Balloon-Helps-Green-Energy-Soar-Sustainably

[7] Rohr S. Heliatek consolidates its technology leadership by establishing a new world record for organic solar technology with a cell efficiency of $12 \%$. Press Release: Heliatek GmbH, Dresden, Germany, January 16, 2013. Online available: http://www.heliatek.com/wp-content/uploads/2013/01/13011 6_PR_Heliatek_achieves_record_cell_effiency_for_OPV.pd f

[8] Y. Gao, T. Martin, E. Niles, A. Wise, A. Thomas, J. Grey. Understanding Morphology-Dependent Polymer Aggregation Properties and Photocurrent Generation in Polythiophene/Fullerene Solar Cells of Variable Compositions. The Journal of Physical Chemistry C. 2010;114(35):15121-8.

[9] Z. Chen,Y.Zheng, H. Yan,A.Facchetti. Naphthalenedicarboximide- vsPerylenedicarboximide-Based Copolymers. Synthesis and Semiconducting Properties in Bottom-Gate N-Channel Organic Transistors. Journal of the American Chemical Society. 2009;131(1):8-9.

[10] C. Brabec, M. Heeney, I. McCulloch, J. Nelson. Influence of blend microstructure on bulk heterojunction organic photovoltaic performance. Chemical Society Reviews. 2011;40(3):1185-99.

[11] F. Yu, H. Wang, S. Zou. Efficient and Tunable Light Trapping Thin Films. The Journal of Physical Chemistry C. 2010;114(5):2066-9. 
[12] L. Huo,J.Hou, S. Zhang, H. Chen, Y. Yang. A Polybenzo[1,2-b:4,5-b']dithiophene Derivative with Deep HOMO Level and Its Application in High-Performance Polymer Solar Cells. AngewandteChemie International Edition. 2010;49(8):1500-3.

[13] S. Price, A. Stuart, W. You. Polycyclic Aromatics with Flanking Thiophenes: Tuning Energy Level and Band Gap of Conjugated Polymers for Bulk HeterojunctionPhotovoltaics. Macromolecules. 2009;43(2):797-804.

[14] R. Mondal,H.Becerril,E.Verploegen, D. Kim, J. Norton,S.Ko, N.Miyaki, S. Lee, M. Toney, J.Brédas,M.McGeheec,Z.Bao. Thiophene-rich fused-aromatic thienopyrazine acceptor for donor-acceptor low band-gap polymers for OTFT and polymer solar cell applications. Journal of Materials Chemistry. 2010;20(28):5823-34.

[15] G. Yu,J.Gao,J.Hummelen,F.Wudl,A.Heeger. Polymer Photovoltaic Cells: Enhanced Efficiencies via a Network of Internal Donor-Acceptor Heterojunctions. Science. 1995;270(5243):1789-91.

[16] Y. Liang,D.Feng, Y. Wu, S. Tsai, G. Li, C. Ray, L. Yu. Highly Efficient Solar Cell Polymers Developed via Fine-Tuning of Structural and Electronic Properties. Journal of the American Chemical Society. 2009;131(22):7792-9.

[17] Y. Liang,Z.Xu, J. Xia, S. Tsai, Y. Wu, G. Li, C. Ray, L. Yu. For the Bright Future-Bulk Heterojunction Polymer Solar
Cells with Power Conversion Efficiency of $7.4 \%$. Advanced Materials. 2010;22(20):E135-E8.

[18] S.Shaheen,R.Radspinner,N.Peyghambarian,G.Jabbour. Fabrication of bulk heterojunction plastic solar cells by screen printing. Applied Physics Letters. 2001;79(18):2996-8.

[19] F. Krebs. Pad printing as a film forming technique for polymer solar cells. Solar Energy Materials and Solar Cells. 2009;93(4):484-90.

[20] C. Hoth,S.Choulis,P.Schilinsky,C.Brabec. High Photovoltaic Performance of Inkjet Printed Polymer:Fullerene Blends. Advanced Materials. 2007;19(22):3973-8.

[21] T. Aernouts,T.Aleksandrov,C.Girotto,J.Genoe,J.Poortmans. Polymer based organic solar cells using ink-jet printed active layers. Applied Physics Letters. 2008;92(3)033306.

[22] C. Hoth,R.Steim,P.Schilinsky, S. Choulis, S. Tedde, O.Hayden, C. Brabeca. Topographical and morphological aspects of spray coated organic photovoltaics. Organic Electronics. 2009;10(4):587-93.

[23] X. Zhao,B.Lojewski, W. Yang, T. Zhu,B.Mi,Z.Gao, W. Huang, W. Deng. Electrospray as a Fabrication Tool in Organic Photovoltaics. Reviews in Nanoscience and Nanotechnology. 2012;1(3):15.

[24] NOAA ESRL Solar Position Calculator, Online Available: http://aa.usno.navy.mil/data/docs/AltAz.php 